\title{
O absenteísmo e suas implicações para o cuidado de enfermagem em serviços de emergência
}

Ione Carvalho Pinto ${ }^{1}$, Brisa Polyana Gonçalves Figueira ${ }^{2}$, Denise Ferro ${ }^{3}$, Fabiana Costa Machado Zacharias ${ }^{4}$, Mariana Figueiredo Souza Gomide ${ }^{5}$, Ricardo Alexandre Arcêncio ${ }^{6}$

\footnotetext{
${ }^{1}$ Enfermeira, Doutora em Enfermagem.

Professora Associada da Escola de Enfermagem de Ribeirão Preto da Universidade de São Paulo. Ribeirão Preto, SP, Brasil. E-mail: ionecarv@hotmail.com.

${ }^{2}$ Enfermeira. Enfermeira do Hospital SírioLibanês. São Paulo, SP, Brasil. E-mail: brisa_polyana@hotmail.com.

${ }^{3}$ Enfermeira, Mestre em Enfermagem em Saúde Pública. Discente do Programa de PósGraduação em Enfermagem em Saúde Pública, nível Doutorado, da Escola de Enfermagem de Ribeirão Preto da Universidade de São Paulo. Ribeirão Preto, SP Brasil. E-mail: denise.ferro@usp.br.

${ }^{4}$ Enfermeira, Mestre em Enfermagem em Saúde Pública. Discente do Programa de PósGraduação em Enfermagem em Saúde Pública, nível Doutorado, da Escola de Enfermagem de Ribeirão Preto da Universidade de São Paulo. Ribeirão Preto, SP, Brasil. E-mail: fabianazacharias@hotmail.com.

${ }^{5}$ Enfermeira, Doutora em Enfermagem em Saúde Pública. Professora Titular do Instituto Taubaté de Ensino Superior. Taubaté, SP, Brasil. E-mail: mariana.souza@usp.br.

${ }^{6}$ Enfermeiro, Doutor em Enfermagem em Saúde Pública. Professor Associado da Escola de Enfermagem de Ribeirão Preto da Universidade de São Paulo. Ribeirão Preto, SP Brasil. E-mail: ricardo@eerp.usp.br.
}

Recebido: 18/03/2016.

Aceito: 19/04/2017

Publicado: 14/07/2017.

Como citar esse artigo:

Pinto IC, Figueira BPG, Ferro D, Zacharias FCM, Gomide MFS, Arcêncio RA. O absenteísmo e suas implicações para o cuidado de enfermagem em serviços de emergência. Rev. Eletr. Enf. [Internet]. 2017 [acesso em:_____ 19:a19. Disponível em http://dx.doi.org/10.5216/ree.v19.40332.

\section{RESUMO}

O estudo buscou identificar a frequência do absenteísmo entre a equipe de enfermagem e seus determinantes em serviços de emergência em saúde. Estudo transversal, realizado por meio de dados secundários dos registros da Coordenação de Recursos Humanos. Identificou-se ocorrências de 208 profissionais de enfermagem, sendo realizada análise univariada, com cálculo de frequência das variáveis idade, sexo, categoria profissional, local de trabalho, dias e motivos do absenteísmo, na sequência, aplicou-se o teste exato de Fisher, fixando-se erro tipo I em 5\%. A média de idade dos participantes foi de 47,2 anos, sendo predominante o sexo feminino (79,8\%). Houve 5.778 ocorrências de absenteísmos (média de 28 por profissional), sendo a doença o principal determinante do absenteísmo (2.671 ocorrências; 46,2\%). O absenteísmo de curto prazo foi predominante (3.020 ocorrências; 52,3\%). Os achados observados nos serviços de emergência são semelhantes, tendo potencial impacto no planejamento, na força de trabalho e qualidade do cuidado prestado.

Descritores: Absenteísmo; Cuidados de Enfermagem; Serviços Médicos de Emergência

\section{INTRODUÇÃO}

A assistência de enfermagem fundamentada em conhecimento, competência e habilidades somente ocorrerá se existirem recursos humanos em termos qualitativos e quantitativos compatíveis à sua execução ${ }^{(1)}$. 
Atualmente, o absenteísmo apresenta-se como um fator determinante e, muitas vezes, como um obstáculo para a gestão em saúde manterem a qualidade do cuidado prestado ${ }^{(2)}$.

O absenteísmo, ou a ausência no trabalho, é considerado um grave problema enfrentado pelos gestores de Recursos Humanos $(\mathrm{RH})$ e refere-se a um afastamento temporário do profissional de suas funções trabalhistas, sendo resultante de inúmeros motivos e trazendo danos para a produção, aumentando os custos da assistência e sobrecarregando outros trabalhadores ${ }^{(3)}$. Conhecer as variáveis que influenciam e determinam o absenteísmo é de extrema importância, tendo em vista que este gera efeito na qualidade do cuidado prestado.

Nos serviços de saúde, a enfermagem é responsável pela composição da maior parte do quadro de funcionários, estando expostos a interações interpessoais constantes, atribuições e exigências de caráter intelectual, além das péssimas condições de trabalho, déficit de $\mathrm{RH}$, insalubridade, jornadas duplas ${ }^{(4-5)}$. Além disso, na enfermagem as condições de trabalho implicam em longas jornadas, em turnos desgastantes, com multiplicidade de funções, ritmo excessivo de trabalho, ansiedade, esforços físicos repetitivos, posições não ergonômicas, resultando em acidentes, doenças e absenteísmo ${ }^{(6-7)}$.

Uma equipe de enfermagem inadequada e o excesso de carga de trabalho tem sido apontado como aqueles que têm impactado de maneira expressiva na ocorrência de eventos adversos no cuidado de enfermagem ${ }^{(8-9)}$. Um quadro de pessoal de enfermagem adequado constitui um indicador de qualidade dos serviços de saúde que exercerá influência nos indicadores assistenciais relacionados aos cuidados de enfermagem ${ }^{(10)}$.

Em uma revisão da literatura orientada pela questão norteadora: "Como o absenteísmo na equipe de enfermagem está sendo abordado pela literatura científica?", foram encontrados artigos que trazem altos índices de absenteísmo em diversos setores da rede hospitalar, principalmente em unidades de terapia intensiva. Além disso, os artigos destacam os motivos que levam os trabalhadores a ausentar-se, independentemente de ausências planejadas ou não planejadas. Apenas dois artigos focalizavam o tema no contexto da rede básica de saúde, o que sugeriu uma lacuna para investigação e necessidade de se buscar novos conhecimentos a respeito do tema.

A literatura tem evidenciado importantes efeitos deletérios à saúde humana decorrentes de um planejamento não adequado, concernente à força de trabalho, dentro dos serviços de saúde. Desta forma, os estudos que possam ajudar na melhoria dos processos de trabalho e na qualidade do cuidado e do cuidar tornam-se cada vez mais necessários ${ }^{(11-12)}$.

Diante do exposto, o objetivo deste estudo foi identificar a frequência do absenteísmo e seus determinantes em serviços de emergência.

\section{MÉTODOS}

Trata-se de um estudo transversal, desenvolvido em município do sudeste brasileiro, que, em 2014, possuía uma população estimada de 658.059 habitantes $^{(13)}$. Na área da saúde, o município encontra-se divido 
em cinco distritos, onde cada um apresenta uma Unidade Básica Distrital de Saúde (UBDS), que por sua vez apresentam um Pronto Atendimento (PA). No ano de 2012 uma UBDS teve seu PA inativado, criando-se assim uma Unidade de Pronto Atendimento (UPA) independente neste distrito.

Os participantes do estudo foram enfermeiros, técnicos e auxiliares de enfermagem, que atuavam em quatro dos cinco serviços de emergência do município, totalizando 208 sujeitos. Adotou-se como critério de inclusão os participantes terem trabalhado nos serviços de emergência no período de janeiro de 2010 a dezembro de 2013. Desta forma, a UPA foi excluída do estudo.

Os dados foram obtidos por meio de documentos e registros da coordenação de Recursos Humanos (RH) dos Distritos e da Secretaria Municipal de Saúde (SMS) concernente ao período de 2010 a 2013. As variáveis levantadas por meio de instrumento previamente formulado foram sexo, categoria profissional, local de trabalho, vínculo empregatício, dias e motivos do absenteísmo.

Quanto ao absenteísmo, este foi classificado segundo as normativas ou aspectos jurídicos legais fornecidos pela organização de saúde investigada, estando essas classificações em: voluntário (qualquer fato que leve o trabalhador a decidir faltar, sem comprovação específica); por doença (toda e qualquer doença que não tenha nexo com o trabalho); patologia profissional (toda e qualquer doença classificada pelo Ministério da Saúde e Ministério da Previdência Social como doença profissional ou relacionada ao trabalho); legal (previstos na legislação trabalhista como férias, licenças, júri, acompanhamento de familiares para tratamento de saúde) e compulsório (representação nacional, afastamento preventivo, prisão em flagrante ou preventiva, greve) ${ }^{(14)}$, como exposto no Quadro 1.

Quadro 1: Classificação do absenteísmo segundo as justificativas ou descrições para as ocorrências. Ribeirão Preto, SP, Brasil, 2010-2013.

\begin{tabular}{|c|c|}
\hline Classificação do Absenteísmo & Justificativa* \\
\hline \multirow{4}{*}{ Voluntário } & Licença Sem Vencimento \\
\hline & Faltas \\
\hline & Falta meio período $(1 / 2)$ \\
\hline & Faltas $1 / 3$ \\
\hline \multirow{10}{*}{ Legal } & Lic. Pessoa família (até 90 dias) \\
\hline & Falta abonada \\
\hline & Disp.ponto(capacit/ev.simil) \\
\hline & Licença gala \\
\hline & Licença nojo \\
\hline & Licença Prêmio em descanso \\
\hline & Licença gestante \\
\hline & Disp.de ponto $\mathrm{p} / \mathrm{serv}$. Estud. \\
\hline & Prorrogação 60 dias licença gestante \\
\hline & Férias \\
\hline Compulsório & Suspensão disciplinar \\
\hline \multirow[t]{3}{*}{ Por Doença } & Art.151, lei 3181/76 - licença tratamento de saúde (primeiros 15 dias) \\
\hline & Art.151, lei 3181/76 - licença tratamento de saúde (a partir do 16ㅇa) \\
\hline & Art.156, lei 3181/76 - licença tratamento de saúde (primeiros 15 dias) \\
\hline \multirow{2}{*}{ Por Patologia Profissional } & Acidente de trabalho (até 15 dias) \\
\hline & Acidente de trabalho (a partir do 16임 \\
\hline
\end{tabular}

Fonte: * RH da Prefeitura Municipal de Ribeirão Preto, 2014. 
Para a análise dos dados fornecidos pela SMS foi realizada a codificação das variáveis e elaborado um dicionário de dados com estas codificações. Estes dados foram digitados em planilha usando o programa Microsoft Excel 2013 e utilizou-se a técnica de dupla digitação, a fim de minimizar erros aleatórios. Procedeuse análise univariada, com cálculo de frequência das variáveis idade, sexo, categoria profissional, local de trabalho, dias e motivos do absenteísmo, na sequência, procedeu-se análise bivariada com cruzamento da variável dependente absenteísmo (motivo do absenteísmo categorizado e/ou dias de absenteísmo) e as variáveis independentes sexo e categoria profissional. Para tal associação, aplicou-se o teste Qui-quadrado, Teste exato de Fisher, com correção de Yates, quando necessários. Para esta etapa, recorreu-se ao Software STATÍSTICA Advanced. Fixou-se o erro tipo I em 5\%.

O estudo foi aprovado pelo Comitê de Ética em Pesquisa da Escola de Enfermagem de Ribeirão Preto, da Universidade de São Paulo, protocolo CAAE: 20588813.0.0000.5393, atendendo assim a Resolução 466/12 do Conselho Nacional de Ética em Pesquisa (CONEP) e outros dispositivos.

\section{RESULTADOS}

Dos 208 participantes do estudo, 167 (80,3\%) eram auxiliares de enfermagem, 29 (13,95\%) enfermeiros e 12 (5,8\%) técnicos de enfermagem. Referente ao sexo, $166(79,8 \%)$, eram do sexo feminino havendo predomínio em todas as categorias; no que tange ao vínculo empregatício, todos os participantes do estudo eram estatutários. A média de idade dos participantes foi de 47,2 anos, com Desvio Padrão (DP) de 9,6 .

Concernente ao local de trabalho, observou-se que, em um dos PA, apenas um enfermeiro atendeu ao critério de inclusão do estudo, que era de permanecer no serviço no período de 2010 a 2013, sugerindo haver uma rotatividade significativa de enfermeiros na referida unidade.

Os resultados mostraram o absenteísmo por doença nos primeiros 15 dias como sendo o de maior frequência, seguido de férias e de faltas injustificadas.

Tabela 1: Frequência de absenteísmo segundo motivo e sexo dos profissionais de enfermagem em serviços de emergência, entre 2010-2013, Ribeirão Preto, SP, Brasil.

\begin{tabular}{ccccc}
\hline Afastamentos & Feminino & Masculino & Total & Valor de $\boldsymbol{p}$ \\
\hline Compulsório & $15(100 \%)$ & & & 0,0001 \\
Por Doença & $1998(74,8 \%)$ & $673(25 \%)$ & $2671(100 \%)$ \\
Legal & $1269(85,1 \%)$ & $222(14,9 \%)$ & $1491(100 \%)$ \\
Por Patol. Profis. & $8(23,5 \%)$ & $26(76,5 \%)$ & $34(100 \%)$ \\
Voluntario & $1160(74 \%)$ & $407(26 \%)$ & $1567(100 \%)$ \\
\hline Total & $4450(77 \%)$ & $1328(23 \%)$ & $5778(100 \%)$ \\
\hline
\end{tabular}

A categoria profissional com maior número de ocorrências de absenteísmo foi a de auxiliar de enfermagem, com frequência de $5.091(88,1 \%$ ) do total de 5.778 ausências nos anos de 2010 a 2013 (Tabela 2). 
Tabela 2: Frequência de absenteísmo segundo motivo e categorias profissionais de enfermagem em serviços de emergência, entre 2010-2013, Ribeirão Preto, SP, Brasil.

\begin{tabular}{|c|c|c|c|c|c|}
\hline Afastamentos & Auxiliar de enfermagem & Enfermeiro & Técnico de enfermagem & Total & Valor de $p$ \\
\hline & & & & & 0,0005 \\
\hline Compulsório & 0 & 15 (100\%) & 0 & $15(100 \%)$ & \\
\hline Por Doença & $2436(91,2 \%)$ & $63(2,4 \%)$ & $172(6,4 \%)$ & $2671(100 \%)$ & \\
\hline Legal & $1153(77,3 \%)$ & $244(16,4 \%)$ & $94(6,3 \%)$ & 1491(100\%) & \\
\hline Por Patol. Profis. & $30(88,25 \%)$ & $0(0 \%)$ & $4(11,75 \%)$ & $34(100 \%)$ & \\
\hline Voluntario & $1472(93,9 \%)$ & $12(0,8 \%)$ & $83(5,3 \%)$ & $1567(100 \%)$ & \\
\hline Total & 5091 & 334 & 353 & 5778 & \\
\hline
\end{tabular}

No que tange aos dias de ausência, independentemente do motivo pelo qual houve este afastamento, as faltas de apenas um dia foram as que se apresentaram em maior frequência, com 3.020 (52,3\%) ocorrências, seguidas das ausências por 15 dias, com 852 (14,75\%) ocorrências, e, posteriormente, seguidas pelos afastamentos de dois dias, com 391 (6,8\%) ocorrências e de 20 dias com 389 (6,7\%) ocorrências.

A Tabela 3 apresenta o número médio de dias de absenteísmo por categoria profissional em relação àqueles que tiveram como justificativa a doença. Observa-se que o absenteísmo de duração curta (um a três dias) foi o mais frequente em todas as categorias profissionais, totalizando 1.763 (66\%) ocorrências de absenteísmo.

Tabela 3: Distribuição das ocorrências de absenteísmo doença segundo número de dias não trabalhados por categoria profissional em serviços de emergência, entre 2011 a 2013, Ribeirão Preto, SP, Brasil.

\begin{tabular}{lllllll}
\hline \multirow{2}{*}{ Dias não trabalhados } & \multicolumn{2}{l}{ Categoria profissional } & \multirow{2}{*}{ Total } & \% & Valor de $\boldsymbol{p}$ \\
\cline { 2 - 4 } & Enfermeiro & Técnico & Auxiliar & & & $>0,0001$ \\
& & & & & & \\
$\mathbf{1}$ a $\mathbf{3}$ & 35 & 143 & 1585 & 1763 & 66,00 & \\
$\mathbf{4}$ a $\mathbf{4}$ & 16 & 26 & 483 & 525 & 19,66 \\
$\mathbf{1 5}$ a $\mathbf{4 5}$ & 12 & 3 & 368 & 383 & 14,34 \\
\hline Total & 63 & 172 & 2436 & 2671 & 100,00 \\
\hline
\end{tabular}

\section{DISCUSSÃO}

Diante do objetivo do estudo de identificar a frequência do absenteísmo, seus determinantes em serviço de emergência e das limitações da utilização de fontes secundárias e do poder de generalização do estudo, observou-se que $97,7 \%$ do total de absenteísmos se deram em decorrência de doença, em que os auxiliares e técnicos de enfermagem são os que apresentam a maior frequência nesta categoria, tais achados são corroborados por diversos estudos ${ }^{(15-17)}$.

Tal fato pode ser analisado devido à divisão do trabalho de enfermagem, em que as referidas categorias desempenham o trabalho predominantemente físico, e também estão mais próximas dos cuidados diretos ao usuário, o que implica em sobrecarga física e emocional mais intensa nestas categorias, com consequência direta em seu adoecimento físico e mental ${ }^{(18)}$.

O sexo feminino foi predominante entre os casos de absenteísmo por doença, o que corrobora com estudo que atribui à própria constituição da categoria profissional, composta majoritariamente pelo sexo feminino. Também é possível acrescentar o fato de que a grande maioria das mulheres apresenta jornada dupla de trabalho, tanto formal quanto informalmente, o que acaba por refletir diretamente na saúde das 
trabalhadoras de enfermagem ${ }^{(16)}$.

Os achados mostram que o absenteísmo doença é composto em sua maioria pelo tipo curta duração (um a três dias), esses resultados são similares aos encontrados em cenário hospitalar no Triângulo Mineiro ${ }^{(19)}$ e podem ser explicados devido ao fato de que, as licenças para tratamento de saúde mais curtas, conforme rege a legislação trabalhista brasileira, não trazem ônus ao trabalhador, tampouco descontos em seu salário e não exigem perícia médica.

Neste trabalho foi possível observar que as faltas injustificadas, que se encontram na categoria de absenteísmo voluntário, apresentam frequência importante de ocorrências com $27,1 \%$ do total de absenteísmos encontrados, tal resultado pode estar relacionado ao fato da amostra de profissionais serem de vínculo estatutário, corroborando com estudos que mostram uma relação positiva entre estas duas variáveis, devido à maior estabilidade profissional atrelada a este tipo de vínculo ${ }^{(17)}$.

O vínculo estatutário pode ser um fator explicativo para a ocorrência de absenteísmo, pelo fato de que a estabilidade é garantida ao servidor público e só poderá ser destituída através de processo administrativo ou judicial, conforme Constituição Federal. Estudos comprovam que servidores públicos se sentem seguros quando ingressam na carreira, sendo que a estabilidade é considerada um dos principais atrativos para se tornar um servidor público, pois gera tranquilidade na vida financeira e social ${ }^{(17,20)}$.

Uma outra questão que paira sobre o estudo é a própria dinâmica organizacional das unidades de saúde, que podem culminar em desmotivações e insatisfação do trabalhador. Além disso, a ausência de planejamento estratégico e da gestão compartilhada entre o enfermeiro e sua equipe de liderados podem potencializar a ocorrência do absenteísmo.

Concomitante ao que foi exposto, o serviço de emergência é uma unidade complexa, que exige da equipe de enfermagem um amplo conhecimento e habilidade tecnológica, bem como, rápido raciocínio e atendimento imediato, o que causa um grande estresse e relações interpessoais difíceis ${ }^{(21)}$. Além disso, é um serviço que funciona $24 \mathrm{~h} / \mathrm{dia}$, com "portas-abertas", recebendo um público grande de pacientes, que, na maioria das vezes não é educado para procurar o serviço de uma forma adequada, gerando sobrecarga de trabalho, baixa qualidade do cuidado prestado e insatisfação profissional ${ }^{(22)}$, ou seja, fatores geradores de absenteísmo.

Os trabalhadores vinculados a SMS do município de Ribeirão Preto não possuem um plano de carreira vigente, sendo que, quando houve a implementação do Pacto pela Saúde, em 2006, uma das exigências era a criação do Plano de Carreira, Cargos e Salário (PCCS) pelos municípios e, quando se trata de satisfação e qualidade de vida no trabalho, é impossível descrevê-las sem correlacionar com o PCCS, pois este incentiva o trabalhador a buscar novos conhecimentos e aprendizados para prestar um cuidado mais qualificado ${ }^{(23)}$. Portanto, a ausência de um plano de carreira no município pode agir como um desestimulante ao trabalhador, fazendo com que este possa ser considerado um fator para o aumento da taxa de absenteísmo nos serviços de emergência estudados. 


\section{CONCLUSÕES}

Assim como no cenário hospitalar, o absenteísmo nos serviços de urgência e emergência apresenta-se como fator preocupante para a qualidade da assistência prestada à população, pois se apresenta em frequências elevadas, e sua maioria é composta pela modalidade de absenteísmo doença, fator agravante quando leva-se em conta as condições de trabalho impostas à equipe de enfermagem e seu adoecimento em decorrência das suas atividades laborais.

Como limitações, por se tratar de um estudo transversal, não é possível a mensuração da relação de causa-efeito, outra questão é que por se utilizar também de dados secundários algumas informações ficaram lacunares, o que pode ter gerado algum viés ao estudo.

O estudo avança no conhecimento ao possibilitar um diagnóstico situacional do absenteísmo no cotidiano da enfermagem em serviços de emergência, trazendo uma questão patente que é o adoecimento dos enfermeiros, o que pode estar relacionado às condições psicológicas e ambientais do cotidiano de trabalho desse profissional, o que exige estudos mais aprofundados para verificar a questão do estresse e outras doenças ocupacionais, especificamente, em áreas que demandam maior sobrecarga de trabalho e mais dispêndio de energia do enfermeiro.

Além disso, o estudo também pode ser utilizado como instrumento de gestão de políticas de RH, considerando que o absenteísmo é um aspecto que necessita ser monitorado com frequência no planejamento de ações que possam ajudar na melhoria dos processos de trabalho, na qualidade do cuidado e na satisfação profissional.

\section{Agradecimento e financiamento}

À Fundação de Amparo à Pesquisa do Estado de São Paulo pelo financiamento do estudo protocolo 2012/51236-1.

\section{REFERÊNCIAS}

1. Da Cruz CW, Bonfim D, Gaidzinski RR, Fugulin FM, Laus AM. The use of Nursing Interventions Classification (NIC) in identifying the workload of nursing: an integrative review. Int J Nurs Knowl. 2014 Oct [acesso em: $17 \mathrm{dez}$ 2016];25(3):154-60. Disponível em: http://doi.wiley.com/10.1111/2047-3095.12031

2. Barbosa JRM, Santana MDR, Araújo AF, Guimarães JMX. Absenteísmo dos profissionais de enfermagem dentro do contexto hospitalar: Revisão integrativa da literatura. Rev e-Ciência. [Internet]. 2016 [acesso em: 17 de 2016];4(1):615. Disponível em: http://dx.doi.org/10.19095/rec.v4i1.81

3. Moraes KN, Ferreira AAR, Fonseca JR, da Silva PLN, Oliveira VV. Fatores relacionados ao absenteísmo por doença em profissionais de enfermagem: uma revisão integrativa. Rev. Eletro. Gestão \& Saúde. [Internet]. 2015 [acesso em: 17 dez 2016];6(1):565-90. Disponível em: http://gestaoesaude.unb.br/index.php/gestaoesaude/article/view/655/pdf 4. Rezende R, Borges NMA, Frota OP. Síndrome de Burnout e absenteísmo em enfermeiros no contexto hospitalar: revisão integrativa da literatura brasileira. Com. Ciênc. Saúde. [Internet]. 2012 [acesso em: 17 mar 2016];23(3):243-52. Disponível em:

http://bvsms.saude.gov.br/bvs/periodicos/revista_ESCS_v23_n3_a6_sindrome_burnout_absenteismo.pdf 
5. Formenton A, Mininel VA, Laus AM. Absenteísmo por doença na equipe de enfermagem de uma operadora de plano de saúde. Rev. Enferm. UERJ. [Internet]. 2014 [acesso em: 17 mar 2016];22(1):42-9. Disponível em:

http://www.e-publicacoes.uerj.br/index.php/enfermagemuerj/article/view/11416/8976

6. Fakih FT, Tanaka LH, Carmagnani MIS. Ausências dos colaboradores de enfermagem do pronto-socorro de um hospital universitário. Acta Paul. Enferm. [Internet]. 2012 [acesso em: 17 mar 2016];25(3):378-85. Disponível em: http://dx.doi.org/10.1590/S0103-21002012000300010

7. Tucker SJ, Harris MR, Pipe TB, Stevens SR. Nurses' ratings of their health and professional work environments. AAOHN J. [Internet]. 2010 [acesso em: 17 mar 2016];58(6):253-67. Disponível em:

http://dx.doi.org/10.3928/08910162-20100526-03Erro! A referência de hiperlink não é válida.

8. Duarte SCM, Stipp MAC, Silva MM, Oliveira FT. Eventos adversos e segurança na assistência de enfermagem. Rev. Bras. Enferm., 2015 [acesso em: 12 dez 2016]; 68(1):144-54. Disponível em: http://dx.doi.org/10.1590/00347167.2015680120p

9. Paiva MCMS, Popim RC, Melleiro MM, Tronchim DMR, Lima SAM, Juliani CMCM. Motivos da equipe de enfermagem para a notificação de eventos adversos. Rev. Latino-Am. Enfermagem, 2014 [acesso em: 12 dez 2016]; 22(5):747-54. Disponível em: www.eerp.usp.br/rlae

10. Versa GLGS, Inaue KG, Nicola AL, Matsuda LM. Influência do dimensionamento da equipe de enfermagem na qualidade do cuidado ao paciente crítico. Texto Contexto Enferm. 2011 [acesso em: 17 dez 2016]; 20(4): 796-802. Disponível em: http://www.textoecontexto.ufsc.br/

11. Vituri DW, Lima SM, Kuwabara CCT, Gil RB, Évora YDM. Dimensionamento de enfermagem hospitalar: modelo OPAS/OMS. Texto Contexto Enferm., 2011 [acesso em: 17 dez 2016];20(3): 547-56. Disponível em: http://www.textoecontexto.ufsc.br/ Erro! A referência de hiperlink não é válida.

12. Mudaly $P$, Nkosi ZZ. Factors influencing nurse absenteeism in a general hospital in Durban, South Africa. J Nurs Manag. [Intermet]. 2015 [acesso em: 17 mar 2016];23(1):623-631. Disponível em:

http://dx.doi.org/10.1111/jonm.12189

13. Instituto Brasileiro de Geografia e Estatística (BR). Estimativa da população de Ribeirão Preto em 10 de junho de 2014. [Internet]. [acesso em: 02 dez 2015]. Disponível em:

http://www.ibge.gov.br/home/estatistica/populacao/estimativa2014/default.shtm

14. Yano SRT, Seo ESM. Ferramenta de coleta de dados para análise do absenteísmo e custo direto para a empresa. INTERFACEHS. [Internet]. 2010 [acesso em: 17 mar 2016];5(2):1-19. Disponível em:

http://www.revistas.sp.senac.br/index.php/ITF/article/viewFile/75/101

15. Magalhães NAC, /v19n2a09.pdf. Farias SNP, Mauro MYC, Donato MD, Domingos AM. O absenteísmo entre trabalhadores de enfermagem no contexto hospitalar. Rev. Enferm. UERJ. [Internet]. 2011 [acesso em 17 mar 2016];19(2):224-30. Disponível em: http://www.facenf.uerj.br/v19n2

16. Campelo CL, Moura LNB, Júnior FJGS, Oliveira FDS, Viana LVM, Silva GP. Absenteísmo na enfermagem: análise do estado da arte. R. Interd. 2016 [acesso em 17 dez 2016]; 9(1): 207-15. Disponível em:

http://revistainterdisciplinar.uninovafapi.edu.br/index.php/revinter/article/view/987/pdf 300

17. Umann J, Guido LA, Freitas EO. Absenteísmo na equipe de enfermagem no contexto hospitalar. Cienc Cuid Saúde. [Internet]. 2011 [acesso em: 17 mar 2016];10(1):184-90. Disponível em:

http://ojs.uem.br/ojs/index.php/CiencCuidSaude/article/viewFile/11867/pdf

18. Silva LGC, Buss AAQ, Haddad MCL, Vannuchi MTO. O absenteísmo da equipe de enfermagem de um hospital universitário público. J. Health Biol Sci. 2016 [acesso em 17 dez 2016];4(2):88-94. Disponível em: doi:10.12662/23173076jhbs.v4i2.520.p88-94.2016

19. Abreu RMD, Simões ALA. Ausências por adoecimento na equipe de enfermagem de um hospital de ensino. Cienc Cuid Saúde. [Internet]. 2009 [acesso em: 17 mar 2016];8(4):637-644. Disponível em:

http://periodicos.uem.br/ojs/index.php/CiencCuidSaude/article/viewFile/9692/5410

20. Fakih FT, Tanaka LH, Carmagnani MIS. Ausências dos colaboradores de enfermagem do pronto-socorro de um hospital universitário. Acta Paul Eferm. 2012 [acesso em: 17 dez2016]; 25(3): 378-85. Disponível em:

http://www2.unifesp.br/acta/

21. Martins JT, Bobroff MCC, Andrade NA, Menezes GD. Equipe de enfermagem de emergência: riscos ocupacionais e medidas de autoproteção. Rev enferm UERJ. [Internet]. 2014 [acesso em: 17 mar 2016];22(3):334-0. Disponível

em: http://dx.doi.org/10.1590/S0034-71672011000500016 
22. O’dwyer G, Matta IEA, Pepe VLE. Avaliação dos serviços hospitalares de emergência do estado do Rio de Janeiro. Ciênc. Saúde Coletiva. [Internet]. 2008 [acesso em: 17 mar 2016];13(5):1637-48. Disponível em: http://dx.doi.org/10.1590/S1413-81232008000500027

23. Lacaz FAC, Vieira NP, Cortizo CT, Junqueira V, Santos APL, Santos FS. Qualidade de vida, gestão do trabalho e plano de carreira como tecnologista em saúde na atenção básica do Sistema Único de Saúde em São Paulo, Brasil. Cad. Saúde Pública. [Internet]. 2010 [acesso em: 17 mar 2016];26(2):253-63. Disponível em:

http://dx.doi.org/10.1590/S0102-311X2010000200005. 